\title{
Damage and Repair Classification in Reinforced Concrete Beams Using Frequency Domain Data
}

\author{
Ali A. Al-Ghalib ${ }^{1, a}$ and Fouad A. Mohammad ${ }^{2, b}$ \\ ${ }^{1}$ Al-Mustansiriya University, Civil Engineering Department, Baghdad \\ ${ }^{2}$ School of Architecture, Design and the Built Environment \\ Nottingham Trent University, Nottingham, NG1 4BU, United Kingdom \\ aali.alghalib@yahoo.com, bfouad.mohammad@ntu.ac.uk
}

Keywords: experimental modal analysis, damage classification, principal component analysis, canonical correlation analysis.

\begin{abstract}
This research aims at developing a new vibration-based damage classification technique that can efficiently be applied to a real-time large data. Statistical pattern recognition paradigm is relevant to perform a reliable site-location damage diagnosis system. By adopting such paradigm, the finite element and other inverse models with their intensive computations, corrections and inherent inaccuracies can be avoided.
\end{abstract}

In this research, a two-stage combination between principal component analysis and Karhunen-Loéve transformation (also known as canonical correlation analysis) was proposed as a statistical-based damage classification technique. Vibration measurements from frequency domain were tested as possible damage-sensitive features. The performance of the proposed system was tested and verified on real vibration measurements collected from five laboratory-scale reinforced concrete beams modelled with various ranges of defects. The results of the system helped in distinguishing between normal and damaged patterns in structural vibration data. Most importantly, the system further dissected reasonably each main damage group into subgroups according to their severity of damage. Its efficiency was conclusively proved on data from both frequency response functions and response-only functions. The outcomes of this two-stage system showed a realistic detection and classification and outperform results from the principal component analysis-only. The success of this classification model is substantially tenable because the observed clusters come from well-controlled and known state conditions.

\section{Introduction}

A large number of technical papers were published over the last three decades on the use of vibration data in the area of Structural Health Monitoring (SHM). Comprehensive literature reviews on methods that have been proposed for civil, aerospace and mechanical structures were cited by Doebling et al. (1996), Doebling et al. (1998), Carden and Fanning (2004) and Sohn et al. (2004). Many methods were achieved tangible success, but the cases were either numerical simulations, or laboratory samples or simulated damage in field structures scheduled for demolition (Friswell and Penny 1997; Carden and Fanning 2004). Therefore, it is widely accepted that there is no algorithm has yet been suggested to generally apply for identifying any type of damage in any type of structure (Carden and Fanning 2004; Carden and Brownjohn 2008; Wenzel 2009). 
Modern SHM combines sensors and computing facilities in real-time vibration-based monitoring systems. The traditional model-based methods, as methods of damage identification, are computationally expensive, error-prone and they are impossible to apply as online real-time diagnostic systems (Wenzel 2009). The programme of a permanent monitoring for suspected structure will end in too many unmanageable data, and therefore Statistical Pattern Recognition (SPR) methods need to introduce as data mining procedure (Wenzel 2009). The damage diagnosis problems in rotating machinery have nearly obtained an exclusive non-model based paradigm (Farrar and Duffey 1999; Farrar and Worden, 2007). However, civil engineering structures do not enjoy the same success of machinery because they are subject to severe operational and environmental conditions (Farrar and Worden, 2007). An early pattern recognition paradigm for SHM of civil engineering structures was first proposed by Sohn and Farrar in 2001 (Sohn and Farrar 2001; Wenzel 2009).

The classification of data from various state conditions is important for effective identification at the quantification and prediction levels (Worden and Dulieu-Barton 2004). Damage detection is also distinguished by making use of the classification step because damage can be identified without previous knowledge on the behaviour of the system when it is damaged (Worden and Dulieu-Barton 2004). Therefore, the classification of the suspected measurements will be the main focus of this study.

PCA as a technique for reduction the dimensionality of a data set was early applied in civil engineering by Sohn and Farrar in 2000 (Farrar et al. 2001; Sohn et al. 2004). In addition, in a technical identification report of applying the SPR paradigm to data from a patrol boat, Sohn et al. (2001) used the PCA method for the discrimination purposes. Nonetheless, despite its reliability as an unsupervised machine learning method for feature extraction, PCA has not been widely tested in the area of the damage identification particularly in civil engineering area.

In this paper, a two-stage combination between Principal Component Analysis (PCA) and Karhunen-Loéve Transformation (KLT) was proposed as a statistical-based damage classification technique. The suggested technique is used to serve as an unsupervised classification tool for data representing different structural conditions. Vibration measurements from frequency-domain were tested as possible damage-sensitive features. Data from both Frequency Response Functions (FRFs) and response-only functions (Response Power Spectra RPS) was acquired and processed from five laboratory-scale Reinforced Concrete (RC) beams modelled with a wide range of common defects.

\section{Classification algorithms}

\subsection{Principal Component Analysis (PCA)}

PCA is a classical technique of multivariate statistics for reduction the dimensionality of a data set into a lower dimension with an insignificant loss of information (Jolliffe 2002). In the SHM context, PCA method is used for two primary applications. First, the technique can be employed as a means of evaluation of patterns in data. The evaluation is achieved through a linear mapping of data from the original feature space into a transformed feature space, where 
the eigenvectors (principal components) are orthogonal to each other. Second, the technique can be used as a tool of reducing the dimensionality of the data. Statistically, the variance in a data matrix $[\mathrm{X}]$ of $(\mathrm{n})$ observations can be expressed as:

$$
[\Sigma]=\frac{1}{n-1} \sum_{i=1}^{n}\left(x_{i}-\bar{x}\right)\left(x_{i}-\bar{x}\right)^{T}
$$

The covariance matrix $[\Sigma]$, which holds the variance of the original data, can linearly be decomposed into a set of orthogonal vectors by using the Singular Value Decomposition (SVD) algorithm, which is given as:

$$
[\Sigma]=[V][\lambda][V]^{T}
$$

Where: $\left[\lambda_{1}\right]$ is a diagonal matrix containing the ranked eigenvalues of $[\Sigma]$ and $[\mathrm{V}]$ is the orthogonal matrix containing the corresponding eigenvectors. The eigenvalues provide information about the variance of the principal components (PCs). The covariance matrix of the new coordinates is a diagonal matrix, and for the first value on the diagonal is to retain the maximum variance. If the $\mathrm{p}$-coordinate data set is completely a linear combination of $\mathrm{r}<$ $\mathrm{p}$ variables, the projections onto the first (r) PCs will entirely describe the data, and the remaining ( $\mathrm{p}-\mathrm{r}$ ) projections or scores will be approximately zero.

\subsection{Karhunen-Loéve Transformation (KLT)}

A general assumption in many forms of PCA as discriminant analysis method is that the covariance matrix $[\Sigma]$ is alike for all groups (the centralisation of data matrix $[\mathrm{X}]$ is based on means of all groups), and the PCA may therefore be done on an estimate of this common within-group covariance. Unfortunately, this procedure probably is not always acceptable for two reasons. First, the within-group covariance matrix may be different between different groups. Second, more serious problem encountered in using PCs based on a unique withingroup covariance matrix to discriminate between groups is that there is no agreement that the separation between groups will be in the direction of the high-variance PCs (Jolliffe 2002). Thus, the first few PCs will only be useful for discriminating between groups in the case where within- and between-group variation have the same dominant directions.

KLT is another statistical multivariate technique related to the PCA family, which is devised to investigate relationships between populations of data sets. In general, KLT in its basic form is similar to PCA. However, the main discrepancy between the two transformations appears in SPR applications because the class information is incorporated in KLT as a supervised learning mode, while it is discarded in PCA (Webb 2002). In the KLT, the new feature space is constructed from eigenvectors of the within-class sample covariance matrix [S], which is defined as (Webb 2002):

$$
[S]=\frac{1}{N-c} \sum_{i=1}^{c} \Sigma_{i}
$$


Where: $\mathrm{c}$ is the number of classes (groups) in data, $\mathrm{N}$ is the number of measurement points (observations) and $\Sigma_{\mathrm{i}}$ is the sample covariance matrix of class (i) group that can be described as:

$$
\Sigma_{i}=\sum_{j=1}^{N}\left(x_{j}-\bar{x}\right)\left(x_{j}-\bar{x}\right)^{T}
$$

The procedure is implemented in two stages. In the first stage, a transformation of data samples to a new space that displays the averaged within-class covariance matrix $[\mathrm{S}]$ as a diagonal matrix. This ensures that the features in the transformed space are uncorrelated, but any further orthogonal transformation will still produce uncorrelated features for which the class-centralised vectors are de-correlated. In this respective, the average within-class covariance matrix in the new transformed space is:

$$
[\Lambda]_{p}=[V]_{p}^{T}[S][V]_{p}
$$

Where: $\left[\Lambda_{1}\right]_{p}$ is a diagonal matrix of the variances of transformed features (eigenvalues of [S]). The first stage of transformation results in a significant reduction of the original data into a new uncorrelated r-dimension space, such that (Webb 2002):

$$
[\Lambda]_{r}=[V]_{r}^{T}[S][V]_{r}
$$

In the second stage of the transformation, the between-class covariance matrix [B] of the original data sets can first be defined as (Webb 2002):

$$
[B]=\frac{c}{(c-1) * N} \sum_{i=1}^{c}\left(x_{i}-\bar{x}\right)\left(x_{i}-\bar{x}\right)^{T}
$$

Where: $\overline{\mathrm{x}}$ are means of features of original data and $\mathrm{x}_{\mathrm{i}}$ are means of the features within the group itself. The between-class covariance matrix [B] is further transformed in a new data space given by the following expression:

$$
[\bar{B}]=[\Lambda]_{r}^{-\frac{1}{2}}[V]_{r}^{T}[B][V][\Lambda]_{r}^{-\frac{1}{2}}
$$

This stage is to compress the class mean information by finding the orthogonal transformation that transforms the class mean vectors into a reduced dimension.

The new definition of data sets in terms of their within-class [S] and between-class [B] covariance matrices leads up to a typical mathematical eigenproblem, where its eigenvalues and eigenvectors can be found by many numerical methods.

\section{Test samples and damage scenarios}

In order to investigate the performance of the proposed classifier on RC structures, five RC beams were constructed and modelled with various damage scenarios. Each beam in its specific state condition was tested under freely supported condition using Experimental 
Modal Analysis (EMA). A photograph representing a generic layout of the EMA set-up is shown in Figure 1.

The five beams were all similar in length and cross-sectional dimensions, but they were different in concrete strength, steel reinforcement and type of damage presented. The five RC beams were cast and tested in the concrete laboratory at Nottingham Trent University, School of Architecture, Design and the Built Environment for the period between October 2010 and October 2011 as a part of the author's PhD programme (Al-Ghalib 2013). The beam names, dimensions and damage configurations are given in Table 1. In addition, Table 2 shows the physical parameters and reinforcement details of the five RC beams.

Table 1: Test RC beams along with the damage conditions.

\begin{tabular}{|c|c|c|}
\hline Test Beam & Dimensions (m) & Damage configuration \\
\hline Beam A & $2.0 \times 0.14 \times 0.09$ & $\begin{array}{l}\text { Tested under seven different state conditions, namely: intact, } 3 \mathrm{kN} \\
\text { load, } 6 \mathrm{kN} \text { load, } 10 \mathrm{kN} \text { load, } 13 \mathrm{kN} \text { load, } 16 \mathrm{kN} \text { load and repaired } \\
\text { conditions }\end{array}$ \\
\hline Beam B & $2.0 \times 0.14 \times 0.09$ & $\begin{array}{l}\text { Tested under seven different state conditions, namely: intact, } 3 \mathrm{kN} \\
\text { load, } 6 \mathrm{kN} \text { load, } 10 \mathrm{kN} \text { load, } 13 \mathrm{kN} \text { load, } 15.5 \mathrm{kN} \text { load and repaired } \\
\text { conditions }\end{array}$ \\
\hline Beam C & $2.0 \times 0.14 \times 0.09$ & $\begin{array}{l}\text { Tested under three different state conditions, namely: intact, } 10 \mathrm{kN} \\
\text { load } 14.5 \mathrm{kN} \text { load conditions }\end{array}$ \\
\hline Beam D & $2.0 \times 0.14 \times 0.09$ & $\begin{array}{l}\text { Tested in two different state conditions; its intact (solid) state } \\
\text { condition and with a purpose-made single void at the end. A void } \\
\text { of } 5 \times 6 \times 40 \mathrm{~cm}^{3} \text { size made of a block of polystyrene was } \\
\text { concealed inside one end of the beam. }\end{array}$ \\
\hline Beam E & $2.0 \times 0.14 \times 0.09$ & $\begin{array}{l}\text { Tested in two different state conditions; its intact (solid) state } \\
\text { condition and with two symmetrical purpose-made voids at the } \\
\text { both ends. Two identical voids each of } 6 \times 10 \times 20 \mathrm{~cm}^{3} \text { size were } \\
\text { concealed inside the right and left ends of the beam. }\end{array}$ \\
\hline
\end{tabular}

Table 2: Specifications of the five test RC beams.

\begin{tabular}{lcccc}
\hline $\begin{array}{l}\text { Test } \\
\text { Beam }\end{array}$ & $\begin{array}{c}\text { Cube strength } \\
\mathrm{N} / \mathrm{mm}^{2}\end{array}$ & Mass kg/m & $\mathrm{E}_{\mathrm{d}} \mathrm{MPa}^{*}$ & Steel Reinforcement \\
\hline Beam A & 20.4 & 2240 & $19.1 \times 10^{3}$ & $2 \varphi 6 \mathrm{~mm}+1 \varphi 10 \mathrm{~mm}$ bottom; $2 \varphi 6 \mathrm{~mm}$ top \\
\hline Beam B & 29.0 & 2270 & $27.5 \times 10^{3}$ & $2 \varphi 6 \mathrm{~mm}+1 \varphi 10 \mathrm{~mm}$ bottom; $2 \varphi 6 \mathrm{~mm}$ top \\
\hline Beam C & 27.3 & 2320 & $25.0 \times 10^{3}$ & $2 \varphi 6 \mathrm{~mm}$ bottom; $2 \varphi 6 \mathrm{~mm}$ top \\
\hline Beam D & 26.0 & 2310 & $24.0 \times 10^{3}$ & $2 \varphi 6 \mathrm{~mm}$ bottom; $2 \varphi 6 \mathrm{~mm}$ top \\
\hline Beam E & 27.3 & 2320 & $27.5 \times 10^{3}$ & $2 \varphi 6 \mathrm{~mm}$ bottom; $2 \varphi 6 \mathrm{~mm}$ top \\
\hline
\end{tabular}

${ }^{*} \mathrm{E}_{\mathrm{d}}$ : Dynamic (tangent) modulus of elasticity.

\section{Description of the induced damage state conditions}

The structural state conditions conducted on the five test beams can be divided into four main groups. The first group was the baseline state condition and referred to as intact for the beams $\mathrm{A}, \mathrm{B}$ and $\mathrm{C}$, but referred to as solid for beams D and $\mathrm{E}$. The second group was concerned only with beams $\mathrm{A}, \mathrm{B}$ and $\mathrm{C}$; this is represented by different levels of flexural cracking resulting from successive four-point bending loadings. The third group was 
interested with studying the effect of repair work conducting on beams A and B only. Finally, the fourth group included defective beams $\mathrm{D}$ and $\mathrm{E}$ where artificial voids were induced at the end of the beams. A photograph for the preparation to cast beam E with two symmetrical voids is shown in Figure 2.

In the case of different levels of flexural cracking, the beams $\mathrm{A}, \mathrm{B}$ and $\mathrm{C}$ were subjected to two symmetrical static point loads at a separation of $0.3 \mathrm{~m}$, as shown in Figure 3.

The beams A, B and C were subjected to incremental load steps to produce controlled levels of damage (see Table 1). At the end of each load step, the beam was unloaded, and the simple supports were replaced by free-free supports to carry out the EMA on a completely freely supported beam (Figure 1). At the final loading step when the beams became too frail, the loading process was stopped and the beams were dynamically tested then repaired. In beam A and beam $\mathrm{B}$, the first visible cracks took place at a load of $10 \mathrm{kN}$, however unlike beam $\mathrm{A}$, in beam $B$ the number of cracks and their length increased substantially with the loading process. At the final stage of the damage scenario, many cracks with a typical length of 40-60 mm spread over more than half the central length of the beam. At the final stage of the damage scenario of beam $C$, many wide open cracks with a typical length of $60-80 \mathrm{~mm}$ spread over more than half the length of the beam. Also, the mid span compression zone of the beam was crumbled, see the photograph below. The cracks went through the bottom face of the beam and displayed crack widths of 1-3 mm under full loading condition. To repair the damage in the three beams, the defective zone was strengthened by bonding a single layer of external carbon fibre reinforced polymer (CFRP) type TORAYCA-FT 300B to cover the defective zone.

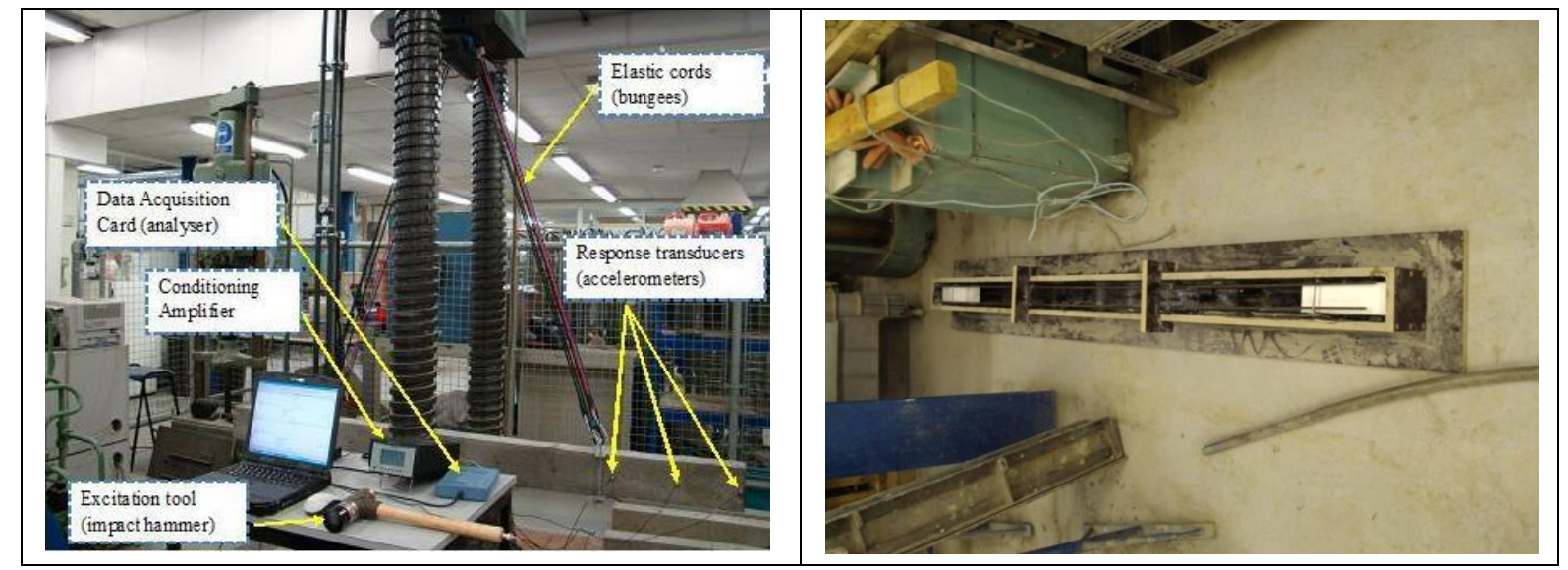

Figure 1: Generic impact hammer modal testing setup.

Figure 2: Beam E- Preparation to cast the beam with two polystyrene blocks. 


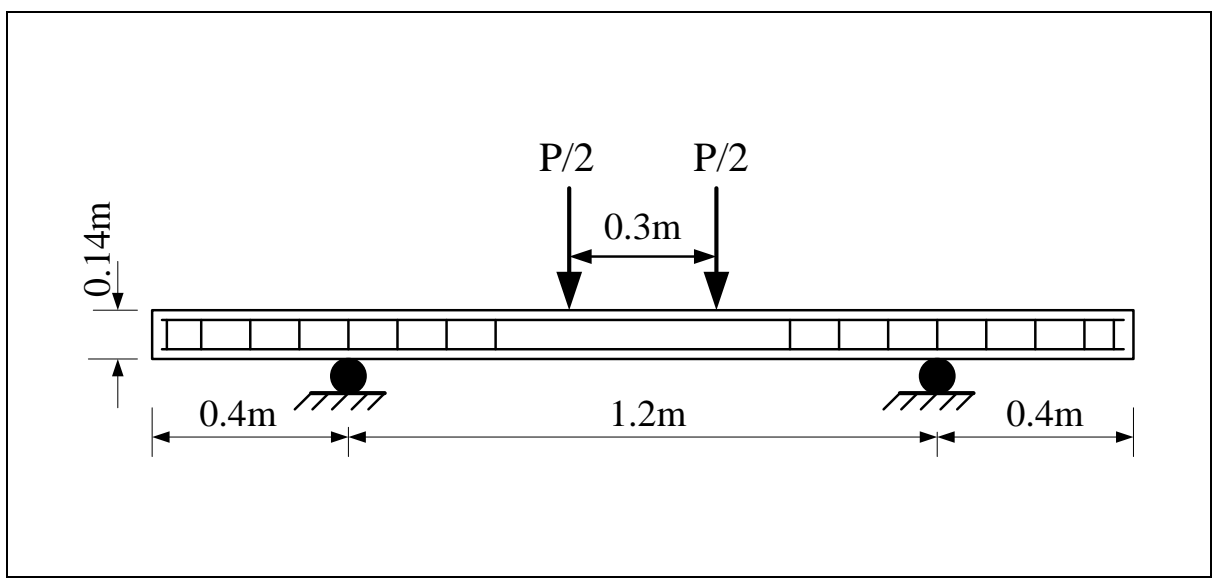

Figure 3: Beam A- Configuration for static loading test.

\section{Laboratory test results}

As an essential step in any EMA, the raw response time signals are transformed into the frequency domain using Fast Fourier Transformation (FFT). Without conducting any normalisation to the time signals, FFT is carried out in order to produce a convenient visualisation to the data in the frequency domain. The sampling parameters selected to digitise the response (acceleration) analogue signals are entirely captured the signal in the recording time period $(\mathrm{T}=500 \mathrm{~ms})$.

Samples of linear response spectra (raw, not averaged) from acceleration time histories of different state conditions at the end point location of beam A are shown in Figure 4. It is obvious from these graphs that the results after $1200 \mathrm{~Hz}$ are neglected, and only data in the frequency range $(0-1200 \mathrm{~Hz})$ is presented. Although the Nyquist frequency corresponding to the sampling rate of this study is $(4090 \mathrm{~Hz} ; \Delta \mathrm{t}=0.1222 \mathrm{~ms})$ higher frequencies are impossible to excite using the current toughest hammer tip. Once the time data is sampled, the linear spectra of the input force and output accelerations are transformed into the frequency domain using the FFT. The non-averaged auto-power input and output spectra alongside the crosspower spectrum between the output and input signals are utilised to obtain the FRF measurements. Samples of the FRFs obtained at $0.6 \mathrm{~m}$ from the beam end for a range of conditions of beam B are given in Figure 5. Researchers are referred to (Al-Ghalib 2013) for more details on the operating, processing and analysis of the vibration measurements.

In the respective of employing FRFs as damage-sensitive features, individual spectra from input and output locations are used to operate the non-averaged populations of FRFs. This procedure is necessary in the context of applying SPR techniques for two major points; to increase the training data sets used to define each condition, and to explore the efficiency of the proposed technique in the presence of data noise. 


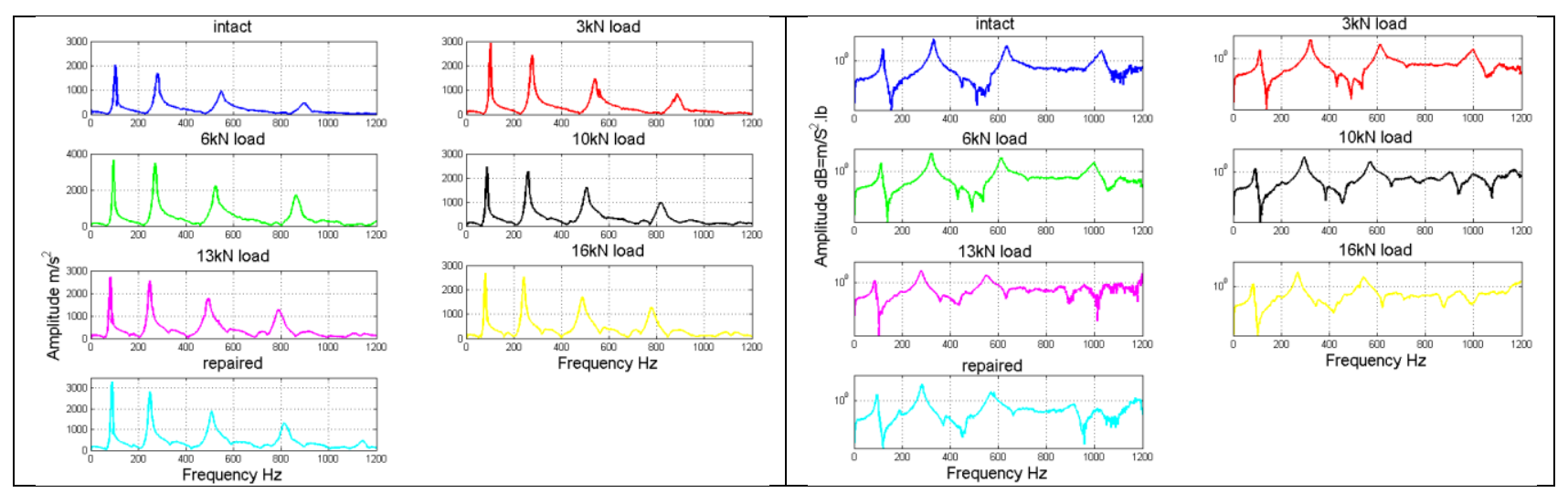

Figure 4: The RPS of beam A at the end point.

Figure 5: The FRFs of beam $B$ at $0.6 \mathrm{~m}$ from the end.

\section{Damage classification using PCA on data sets from frequency domain}

With a view to assess the performance of PCA as damage classification tool, FRF data sets as damage-sensitive features were examined in this section. Although FRF as a damagesensitive feature entails further computational effort compared with the time histories data, FRF explains the relationship between the input and the response; therefore it completely describes the structural dynamic properties (Maia et al. 1997; Figueiredo et al. 2009). As a preparation effort for the discriminant analysis using PCA, the feature-vectors matrix was assembled by using the amplitude of the FRFs with frequency range $0-1200 \mathrm{~Hz}$. Consequently, the sets for the analysis and graphical illustration were aggregated as in the following steps:

- The training-set matrix ( $\mathrm{n} \times \mathrm{p}$ ) of FRFs was piled from all structural conditions corresponding to the beam case study. For beam A and beam B, the state conditions were divided in (7) groups, intact, five different damage levels and repaired beam. For beam $\mathrm{C}$, the state conditions were three, intact and two severely damaged cases. Finally, for beams $\mathrm{D}$ and $\mathrm{E}$, the conditions were two, the solid beam and beam with void(s).

- The rows (n) of the training feature matrix represent the frequency data points (600). The columns (p) represent the features related to the beam and their number varies according to the particular beam case study.

- For beams A and B, the number of feature vectors ( $\mathrm{p})$ was $(44 \times 7)$ resulting in data from all channels with four repetitions for each sensor. In beam $C$, the number of the feature vectors was $(55 \times 3)$ with five repetitions for the (11) measurement points for the three cases. Finally, in beams D and E, (p) was $(44 \times 2)$.

- The global training matrix was normalised, and the corresponding covariance matrix $[\Sigma]$ was obtained from Equation 1. The covariance matrix of each beam case study was transformed to the uncorrelated feature space conducted by the SVD, and the first two PCs were plotted, accordingly. Each feature vector in the $[\Sigma]$ arose as a dot in the first two PCs plot. 
Figure 6 shows the first two PCs of all the five beams analysed for this task. In addition, the corresponding algorithm that performs PCA is given in the upper part of the flow chart of Figure 7, and specified as unsupervised learning mode. Having examined the FRFs as damage-sensitive feature in conjunction with the unsupervised PCA method, the analysis manages to separate qualitatively feature vectors from various groups into two main groups. In principal, the first two PCs provide a general distinction between undamaged states and damaged states without deep dissection for features from different levels included in each main group. However, this discriminant analysis does not tend to further dissect every main cloud of data into its original subgroups. There are signs of local congregation of data from closer levels of loading can be recognised when each cloud is scrutinised carefully. Providentially, features of the same type of underlying damage condition arise close to each other, when visualised by the first two PCs. For the test beams A, B and C, as data from these samples includes various multiple conditions, PCA indicates the incapacity to provide more than two groups (Figure 6).

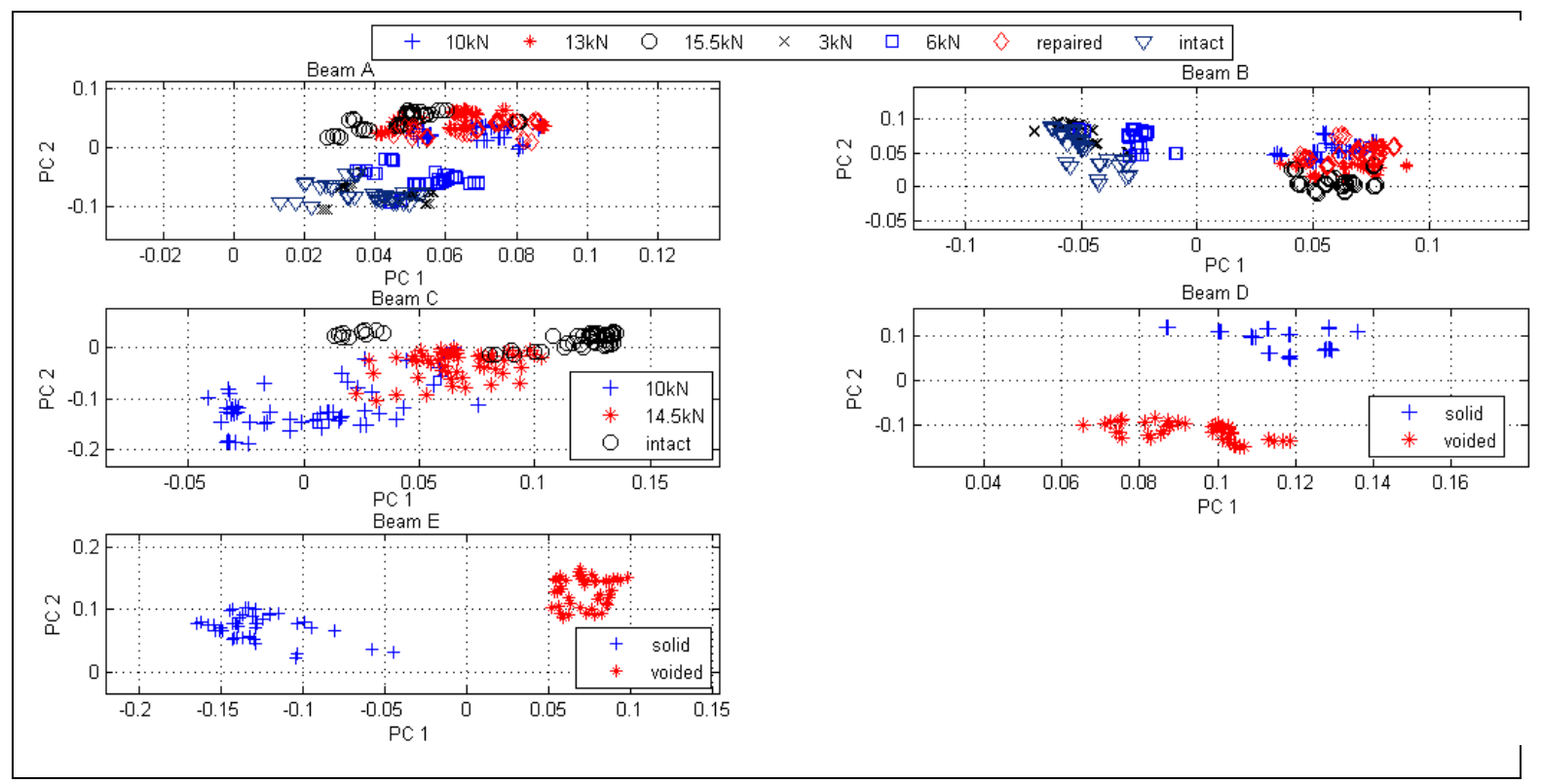

Figure 6: First and second PCs based on data sets of FRFs, all five beams. 


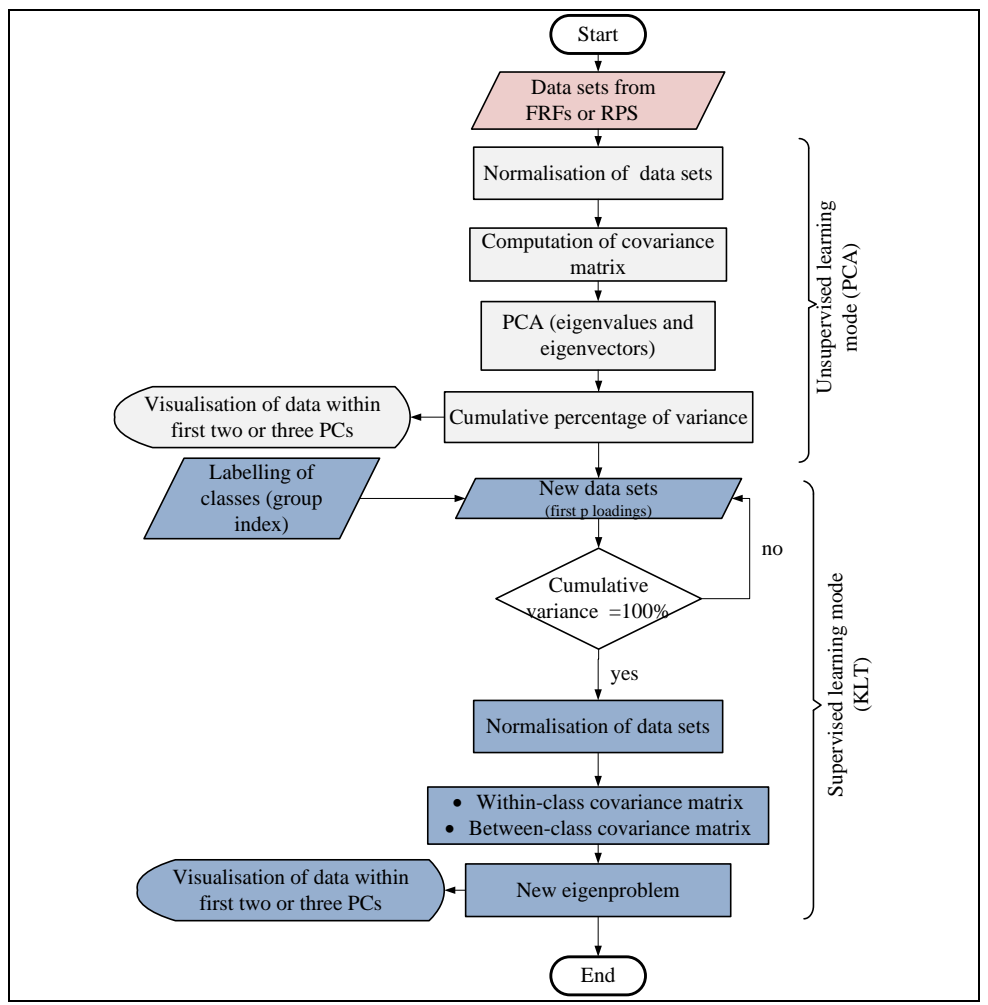

Figure 7: Flow chart of the proposed classification paradigm.

\section{Damage classification using the proposed PCA-KLT on data sets from FRFs}

In order to establish more sensitive classification means, KLT technique is suggested to apply at data from frequency domain. The proposed technique integrates the first stage of the PCA, and uses the output data from the PCA transformation as input data set for the second transformation stage. The lower part of the flow chart given in Figure 7 presents the main steps of the second transformation stage. The proposed classification technique can be outlined in the following steps:

- A number ( $\mathrm{r}$ ) of the first loading vectors (eigenvectors) from the first transformation stage (PCA) that account for almost $100 \%$ of the total variation are set to be the input data matrix for the KLT.

- The rows of the new feature vectors (p) must be labelled according to their respective classes.

- The within-class covariance matrix (S, Equation 3) and between-class covariance matrix (B, Equation 7) are computed using the new set of data. Both covariance matrices are decomposed into new uncorrelated space.

- The plot of the first two PCs, from the new transformation, will offer insight to the visualisation of the data. Valuable amount of the information in data is maximised and inserted in the first two or three PCs. The first few PCs account for higher percentage of the total variation, and no significant loss in information is experienced.

For a comparison purpose, the same data sets used in PCA (section 7) were utilised also in the proposed classification system. In the new system, $100 \%$ of the total variation is held by 
the first five PCs for beams A and B, and in the first two PCs for beam C. Most interestingly, Figure 8 shows the results from the proposed system based on the standardised data of FRFs for test beams A, B and C. For beams A and B, the plots identify four groups of data, corresponding to the seven state conditions, suggesting that data from (intact, $3 \mathrm{kN}$ ), $6 \mathrm{kN}$, $(10 \mathrm{kN}$, repaired) and $(13 \mathrm{kN}, 16 \mathrm{kN})$ are four distinctive different groups. The overlap between cluster $(13 \mathrm{kN}, 16 \mathrm{kN})$ is minimal in beam A and beam B compared with the joint clusters of other state conditions. For beam $\mathrm{C}$, three clear clusters, corresponding to the three state conditions, are identified without any interference between the groups. Though, the data per each group of this case is more dispersive compared with the previous two beams. The data used to plot Figure 8 accounts for $71 \%, 70 \%$ and $100 \%$ of the total variance in beams A, B and $\mathrm{C}$, respectively. This suggests that the first two components in beam $\mathrm{C}$ and the first three components in beams $\mathrm{A}$ and $\mathrm{B}$ give better representation to the data compared with similar components of PCA.

\section{Damage classification using the proposed PCA-KLT on data sets from RPS}

In the context of using RPS densities as damage-sensitive feature, Figure 9 displays the first two component plots based upon the proposed transformation method, and on data from RPS density. In this context, the first two PCs hold $93 \%, 78 \%$ and $100 \%$ of the total variance for beam A, beam B and beam C, respectively. This suggests that data from RPS density is more convenient to be represented by first two PCs than data from FRFs. The first two components from transformed space of RPS as damage-sensitive feature provide better approximation for the data; hence, the visualisation defines the groups objectively. For beams A and B, the graphical illustration recognises four distinctive groups, related to the seven cases, suggesting that data from (intact, $3 \mathrm{kN}), 6 \mathrm{kN},(10 \mathrm{kN}$, repaired) and $(13 \mathrm{kN}, 16 \mathrm{kN})$ are separate four groups. Most importantly in the classification context, separation process always places the data of intact and barely damaged groups far away from data of severely damaged beams. Also, this is the case in beam $\mathrm{C}$, where three groups are clearly isolated in the meantime the data per each group is less dispersive compared with the groups in Figure 8.

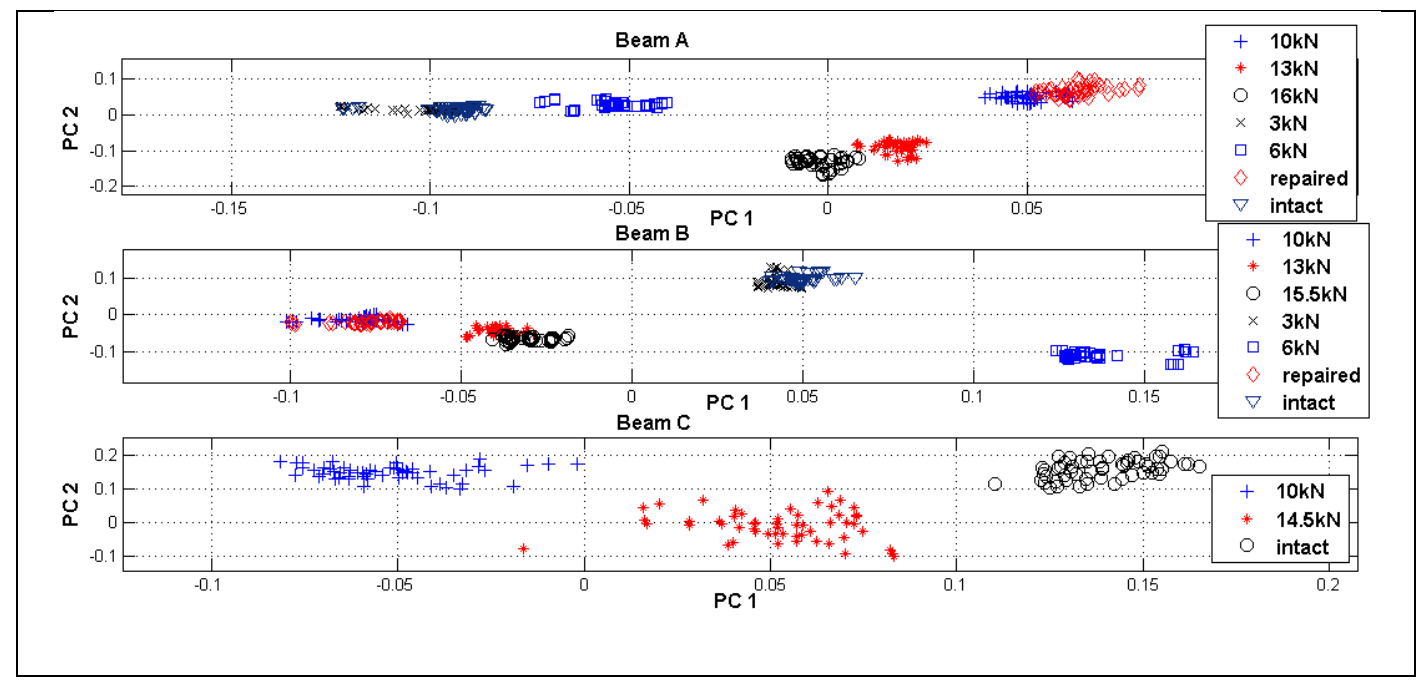

Figure 8: First and second PCs of KLT based on data from FRFs. 


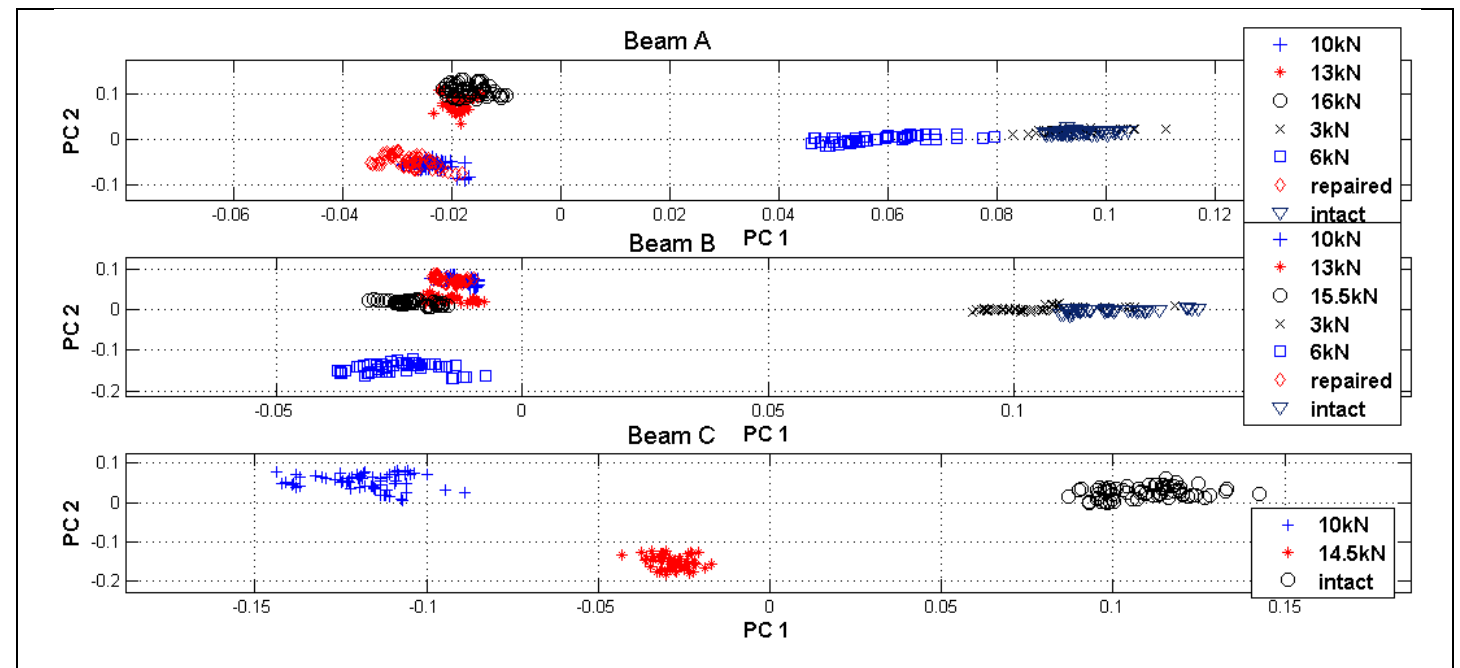

Figure 9: First and second PCs of KLT based on data from RPS.

\section{Conclusions}

Based on the classification results of PCA analysis (Figure 6), the most interesting finding is that two clouds of data are visually distinct in all the five beams. This sensible separation is done by the first two PCs and carried out in an unsupervised mode. The visual discrimination holds fairly well between data from intact and lightly damaged and data from severely damaged conditions. However, this discriminant analysis does not tend to further dissect every main cloud of data into its original subgroups. There are signs of local congregation of data from closer levels of loading can be recognised when each cloud scrutinised carefully. On the other hand, the plots of the first two PCs, from the new transformation (Figures 8 and 9), offer insight to the visualisation of the data. Valuable amount of the information in data is maximised and inserted in the new first two PCs. The first two PCs from PCA-KLT model account for higher percentage of the variance of data sets compared with the PCA method alone. Therefore, the visualisation of the first two PCs discriminates qualitatively between the subgroups of each major state condition group. The same data that classified into two major groups by the PCA method is further dissected into five distinctive subgroups by using the suggested PCA-KLT method. Finally, although the vibration measurements of this study were conducted by using the impact hammer excitation, nothing prevents the proposed classifier from being used with other excitation tools.

\section{References}

Al-Ghalib AA. (2013), Damage and Repair Identification in RC Beams Modelled with Various Damage Scenarios Using Vibration Data, PhD. Thesis, Nottingham Trent University, Nottingham, UK.

Carden EP., Fanning P. (2004), Vibration Based Condition Monitoring: A Review. Journal of Structural Health Monitoring 3(4), 355-377. 
Carden P., Brownjohn J. (2008), ARMA Modelled Time-Series Classification for Structural Health Monitoring of Civil Infrastructure. Mechanical. Systems and Signal Processing 22, pp. 295-314.

Doebling S., Farrar C. and Prime M. (1998). A Summary Review of Vibration-Based Damage Identification Methods. The Shock and Vibration Digest, 30(2), pp. 91-105.

Doebling SW., Farrar CR., Prime MB. and Shevitz DW. (1996). Damage Identification and Health Monitoring of Structural and Mechanical Systems from Changes in Their Vibration Characteristics: A Literature Review. Technical Report LA-13070-MS, Los Alamos National Laboratory, Los Alamos, NM, USA.

Farrar C. and Duffey T. (1999). Vibration Based Damage Detection in Rotating Machinery and Comparison to Civil Engineering Applications. Proceedings of the 3rd International Conference on Damage Assessment of Structures, DAMAS'99, Dublin, Ireland.

Farrar C. and Worden K. (2007). An Introduction to Structural Health Monitoring. Philosophy Transactions of Royal Society, Vol.365, pp. 303-315.

Farrar C., Doebling S. and Nix D. (2001). Vibration-Based Structural Damage Identification. Philosophy Transactions Royal Society, Vol. 359, pp. 131-149.

Figueiredo E., Park G., Figueriras J., Farrar C., Worden K. (2009), Structural Health Monitoring Algorithm Comprises Using Standard Data Sets. Report LA-14393, Los Alamos National Laboratory, Los Alamos, New Mexico.

Friswell MI., Penny JET. (1997), Is Damage Location using Vibration Measurements Practical? Structural Damage Assessment using Advanced Signal Processing Procedures, Proceedings of DAMAS '97, Sheffield, UK,351-362.

Jolliffe IT. (2002), Principal Component Analysis, 2nd ed., Springer, Verlag, New York. Maia NMM., Silva JM., He J., Lieven NAJ., Lin RM., Skingle GW., To W-M. and Urgueira APV. (1997). Theoretical and Experimental Modal Analysis. Editors: Maia and Silva, UK: Research Studies Press Ltd., Taunton, Somerset, England.

Sohn H. and Farrar CR. (2001). Damage Diagnosis using Time Series Analysis of Vibration Signals. Journal of Smart Material and Structures, Vol. 10, pp. 446-451.

Sohn H., Farrar CR., Hemez FM., Shunk DD., Stinemates DW., Nadler BR. and Czarnecki JJ. (2004). A Review of Structural Health Monitoring Literature: 1996-2001. Technical Report LA-13976-MS, Los Alamos National Laboratory, Los Alamos, NM, USA.

Webb A. (2002), Statistical Pattern Recognition. 2nd ed., John Wiley \& Sons, West Sussex. Wenzel H. (2009), Health Monitoring of Bridges. John Wiley \& Sons, West Sussex.

Worden K., Dulieu-Barton JM. (2004). An Overview of Intelligent Fault Detection in Systems and Structures. Journal of Structural Health Monitoring, Vol. 3(1), 085-098. 